\title{
DELEUZE, COLOR Y COSMOS-KLEE ${ }^{1}$
}

\author{
Sergio Martinez $V^{2}$
}

\begin{abstract}
RESUMEN: En este trabajo buscamos proponer una teoría del color de inspiración deleuzeana, esto es, propondremos una teoría que atenderá al color en cuanto multiplicidad y acontecimiento. Para ello, ensayaremos un recorrido a través de ciertos pasajes deleuzeanos que nos permitirán avanzar en dicha propuesta y llevarla al territorio artístico para establecer una interferencia que implicará variar la dirección de nuestro trabajo al proponer un diálogo con Paul Klee. En definitiva, intentaremos desarrollar las siguientes preguntas: ¿Cómo pensar una teoría del color de inspiración deleuzeana? ¿Cómo llevarla al terreno artístico? ¿Cómo concebir su relación con un cosmos-Klee? Y esto, para intentar establecer a través de una conversación que podría sostenerse con la obra de Paul Klee otra perspectiva de trabajo en torno a la obra de Gilles Deleuze.
\end{abstract}

Palabras Clave: Deleuze. Klee. Color. Multiplicidad. Acontecimiento. Artes.

Estableciendo un parámetro para aproximarnos a una teoría del color de inspiración deleuzeana, esto es, considerándola esencialmente relacionada a acontecimientos para concebir su conceptualización, podríamos atender a una idea que exprese la realidad del color; es que al entrar a un dominio de corte deleuzeano debemos tomar la precaución de considerar dicha idea en cuanto problemática, porque si el color $^{3}$ es en principio un acontecimiento su

1 http://dx.doi.org/10.1590/S0101-31732018000100011 Agradezco a Miguel Ruiz Stull unas conversaciones en torno a este trabajo.

2 Universidad de Chile, Facultad de Artes, Santiago, Chile. E-Mail: sergio.sermar@gmail.com

Doctor (C) Filosofía. Mención Estética y Teoría del Arte. Becario Conicyt 2013-2017. Áreas de interés: filosofía moderna (I. Kant, F. Nietzsche), filosofía contemporánea (G. Deleuze), arte moderno (P. Klee).

3 Nos remitimos al capítulo “QQué es un concepto?” de ¿Qué es la filosofía? (2005). Las definiciones que propone Deleuze-Guattari para describir qué es un concepto podrían perfectamente remitir, por el tenor de la pregunta "qu'est-ce qu'un concept?" y, como veremos, por la variación que propone Deleuze de la noción de esencia, a la concepción de Idea problemática elaborada en el cuarto capítulo de Diferencia y repetición (2011) titulado: Sintesis ideal de la diferencia. Ahora bien, al considerar esta remisión, que podría permitirnos equiparar ambas operaciones y, al mismo tiempo, no confundirlas al ser relacionables en sus usos, es la noción de concepto la que no podría ser concebible en términos de identidad. Esto es, concepto (en ¿Qué es la filosofía?) e Idea (en Diferencia y repetición) remiten a la elaboración de una noción de acontecimiento que bien implica una heterogeneidad inasimilable a la diversidad sensible que la actualiza. Si, en los términos de François Zouravichvilli, no es menor el ejemplo, "“." [e]l concepto de comienzo no envuelve a la unicidad sino a condición de presuponer la

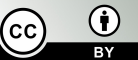

This is an open-access article distributed under the terms of the Creative Commons Attribution License. 
actualización no agota su realidad; y si un concepto de color toma consistencia en un plano de inmanencia, el campo al que este remitiría habríamos de considerarlo trascendentalmente. ${ }^{4}$ ¿Cuál es la naturaleza de este acontecimiento? esto es lo que intentaremos avanzar en este trabajo. Un avance que quizá habríamos de considerar como un paso lógico, si consideramos que la lógica en tanto que construcción de un sentido y exploración de una sensación implica una heterogeneidad irreducible a los principios que ella misma presupondría. Es decir, considerando que una lógica implica una génesis heterogénea que actúa dinámicamente, un principio, si mantenemos esta noción no-deleuzeana, no podría sino ser diferencial en su esencia. Y bien podríamos atender esta vieja noción si consideramos lo que el mismo Deleuze nos dice de ella, porque

Los acontecimientos y las singularidades de la Idea no dejan subsistir ninguna posición de la esencia como $<<$ lo que la cosa es $>>$. Y, sin duda, se permite conservar la palabra esencia, si se tiene interés en ello, pero con la condición de decir que la esencia es precisamente el accidente, el acontecimiento, el sentido. (DELEUZE, 2012, p. 290/2011, p. 247-248).

identidad de lo que se ha de pensar. Veremos que el comienzo debe ser repetido, e incluso afirmado «todas las veces», porque el mundo no tiene la realidad o la fiabilidad que creemos: es heterogéneo. Al mismo tiempo que el pensamiento afirma una relación absoluta con la exterioridad, recusa el postulado del reconocimiento y afirma el afuera en este mundo: heterogeneidad, divergencia. Cuando la filosofía renuncia a fundar, el afuera abjura de su trascendencia y se vuelve inmanente. [...]. El verdadero comienzo está necesariamente fuera-del concepto, o en el límite del concepto, y depende de la capacidad de este último para no cerrarse sobre sí, para implicar, al contrario, la relación con el afuera de la que extrae su necesidad. Podemos prever ya que esta relación pondrá en juego algo muy distinto de una «realidad exterior» (un acontecimiento, un devenir)." (ZOURABICHVILI, 2004, p. 26-27).

${ }^{4}$ Respecto a la relación entre Kant y Deleuze, creemos que al menos habría que considerar que esta se inscribe y elabora bajo consideraciones genéticas (para Deleuze, solo en la tercera crítica es cuando Kant propone un argumento genético que da cuenta específicamente de la génesis de las facultades) que problematizan tanto el estatuto de la Idea y el ser de lo sensible (cuarto y quinto capítulo de Diferencia y repetición, 2011) como la presuposición kantiana de un sujeto de conocimiento análogo a la experiencia empírica dada, lo que conlleva replantear la estética trascendental. Implicado en esto último está al menos: 1) la necesidad de problematizar la sensación: esta no está dada y es necesario preguntar por su génesis y, ello conlleva considerar que el espacio se compone de forma paulatina, es decir, 2) la receptividad del yo pasivo: en Kant la síntesis se reserva a la actividad y Deleuze concibe una síntesis pasiva operando en la individuación del individuo, 3) el tiempo como determinante de la relación entre actividad y pasividad: sus tres operaciones sintéticas, enfatizando la tercera síntesis del tiempo: la síntesis estática del tiempo (la del orden del tiempo) en cuanto ella expresa el giro trascendental. En torno al empirismo trascendental, o al campo trascendental pre-individual y presubjetivo concebido como un proceso de diferent/ciación, propuesto por Deleuze: L. Bryant (2009), G. Montenegro (2013, capítulos II y III), A.Sauvagnnargues (2009a, capítulos I, II y IV), D. Voss (2013). Además, cabe mencionar los artículos de: D. W. Smith (1997, p. 29-56), M. Rölli (2009, p. 26-53), B. Lord (2012, p. 82-102), M. Ruiz Stull (2013, p. 53-64). 
Si una multiplicidad virtual es una Idea, ¿el atender a lo que la determina como tal, a la diferencia que la torna actual, qué implicancias podría tener para pensar en una teoría del color de inspiración deleuzeana? ¿podríamos acaso extraerla del terreno artístico? ¿cómo concebir su relación con un cosmos-Klee?

\section{I}

En La concepción de la diferencia en Bergson, Deleuze, entre otros casos, alude a la noción de color para pensar en el concepto de naturaleza que se encuentra en el filósofo francés. Y esto es fundamental si atendemos a lo que se juega en dicho texto, leer a Bergson como un pensador de la diferencia ontológica.

Esta filosofía [la bergsoniana] opera siempre en dos planos, metodológico y ontológico. Por una parte, trata de determinar las diferencias de naturaleza entre las cosas: solamente así podremos <<volver >> a las cosas mismas y dar cuenta de ellas sin reducirlas a algo distinto, captarlas en su ser. Pero, por otra parte, si el ser de las cosas reside en cierto modo en sus diferencias de naturaleza, podemos esperar que la misma diferencia sea alguna cosa, que tenga una naturaleza que nos entregue el Ser. Estos dos problemas, el metodológico y el ontológico, remiten constantemente el uno al otro: diferencias de naturaleza y naturaleza de la diferencia. En Bergson, aparecen ligados, y encontramos los caminos que los comunican. (DELEUZE, 2005, p. 45/2002, p. 43).

Si al intentar concebir a la vez: diferencias de naturaleza y naturaleza de la diferencia, consideramos que una de las tareas que el binomio BergsonDeleuze le imponen a la filosofía no es otra que hallar para cada caso un concepto adecuado que nos permita dar cuenta de la singularidad de él, podríamos quizá llegar a expresar cuál es la singularidad de la naturaleza del color. Atendamos, entonces, a lo que Deleuze nos propone en dicho texto. Pues si un concepto de color implica una multiplicidad de colores a experimentar, habría de considerar que las diferencias de naturaleza entre los colores son inmanentes al devenir de su propia naturaleza. Y Deleuze (2005, p. $59 / 2002$, p. 60) resalta que el caso de los colores provisto por Bergson atiende la génesis del color, esto es, la diferencia que actualiza los matices, las relaciones componentes de dicho concepto. Un movimiento, desde la divergencia entre los colores hacia la convergencia en un mismo punto, y viceversa. Como dirá Bergson, en el texto al que nos remite Deleuze (2005, p. 59/2002, p. 60), esto 
[c] onsistiría aquí en tomar los mil matices del azul, del violeta, del verde, del amarillo, del rojo, y, haciendo que atraviesen una lente convergente, llevarlos sobre un mismo punto. Entonces aparecería en todo su esplendor la pura luz blanca, aquella que, percibida aquí abajo en los matices que la dispersan, encerraba allí arriba, en su unidad indivisa, la diversidad indefinida de los rayos multicolores. Entonces se revelaría también, hasta en cada matiz tomado aisladamente, lo que el ojo en principio no notaba, la luz blanca de la que participaba, la iluminación común de donde extrae su coloración propia. (BERGSON, 2013, p. 255-256).

En los términos de Deleuze (2005, p. 59-60/2002, p. 60-61), “[C] uando las cosas se convierten en matices del concepto", "[l]as diferencias espaciales se han sustituidos por diferencias temporales. [Y] es propio de la diferencia temporal el hacer del concepto una cosa concreta, porque las cosas son matices o grado que se presentan en el seno del concepto". Esto es, cuando los colores convergen virtualmente en la pura luz blanca, las diferencias que componen dicho concepto de color no solo expresan una cosa concreta, pues también lo que deviene actual por un movimiento de color, de la pura luz blanca a la diversidad indefinida de los colores, es la naturaleza temporal propia a la de la duración. Ahora bien, "la diferencia" no consiste sino en operar diferencialmente entre: unidad indivisa de la luz y diversidad indefinida de los colores, o sea, ella, virtual/actual en su operación, no solo está determinada por el tiempo sino también por un movimiento cuya duración no puede sino concebirse en alteración. ${ }^{5}$ Esto es, nunca se trata del mismo movimiento, nunca

\footnotetext{
${ }^{5}$ La tesis de la mismidad del movimiento ha sido problematizada, sosteniendo un encuentro con la filosofía deleuzeana, por Miguel Ruiz S. en un extraordinario libro titulado: Tiempo y experiencia. Variaciones en torno a Bergson (2013). Notablemente, en el capítulo tercero: Vida, multiplicidad y actualidad, se nos propone una lectura del concepto de alteración (alloiosis), extraído de la física aristotélica, que nos vuelve a plantear la naturaleza del movimiento bajo consideraciones bergsonianas. Pues si el movimiento ya en el espacio no puede ser concebido bajo términos de traslación (phora) es al estar implicado el tiempo en una noción no extensiva del movimiento (y viceversa) que las cualidades de este son distintas al número que al describirlas cuantitativamente lo determinan formalmente. Si el tiempo, como diría Deleuze en alusión a Hamlet, está fuera de sus goznes, se ha liberado del número que se le adscribía, ahora el movimiento físicamente distinto a la unidad numérica que podría llegarse a atribuírsele puede llegar a ser concebido hetero-genéticamente. Más, pues si la naturaleza del movimiento se plantea como un mixto de duración/materia, esto es, en su heterogeneidad y acontecimiento, la eficacia del tiempo en aquel cobra suma relevancia al ser concebible la realidad no-numérica del movimiento virtual y actual en términos de alteración (en este sentido, la naturaleza del número deviene numerante). En otros términos, como nos lo hace saber Ruiz S., es posible pensar una heterogeneidad propia a la relación entre física y matemática que puede esencialmente pensarse como una variación entre magnitudes no-numéricas diferencialmente. Y ello implica concebir la composición o complejidad de lo real, sus relaciones, en términos de multiplicidad. Como alteración
} 
se trata del mismo color, se trata de un cambio cualitativo, de lo que difiere de sí mismo: el concepto de color concibe los matices que lo definen implicados en él mismo. Un movimiento que al ser concebible cuantitativamente es extensible espacialmente, pero que al ser pensable cualitativamente, aun si la cantidad puede explicar la cualidad y el tiempo concebirse espacialmente, ya no nos remitiría meramente a su actualidad sino también a su virtualidad. Como dirá Deleuze (2005, p. 61/2002, p. 62), "Lo virtual define ahora un modo de existencia absolutamente positivo. La duración es lo virtual; es real tal o cual grado de la duración en la medida en que ese grado se diferencia”. La duración propia del color podría ser pensada cuando consideramos que sus diferencias de naturaleza se deben a que "[...] lo que se diferencia es desde el principio lo que difiere de sí mismo, es decir lo virtual. La diferenciación no es el concepto, sino la producción de los objetos de los cuales da razón el concepto." (DELEUZE, 2005, p. 59/2002, p. 60). En una palabra, un color al diferenciarse va produciendo determinados matices que están implicados virtualmente en un movimiento propio a dicho concepto que habría de concebir en términos de acontecimiento.

Ahora bien, hemos dicho que Deleuze hallaba en Bergson una concepción de la diferencia en la que se entrelazaban cuestiones metodológicas y ontológicas, y si estamos intentando considerar diferencias de naturaleza $y$ la naturaleza de la diferencia de un color, podríamos considerar que dicha concepción de la diferencia busca atender al movimiento que se establece entre lo virtual y lo actual. Pues bien, si en Diferencia y repetición nos propondrá

Retomemos el movimiento de la Idea, inseparable de un proceso de actualización. Una idea, una multiplicidad, como la de color [comme celle de couleur] [cursivas nuestras], por ejemplo, está constituida por la coexistencia virtual de las relaciones entre elementos genéticos o diferenciales de un cierto orden. Son esas relaciones las que se actualizan en colores cualitativamente distintos, al mismo tiempo que sus puntos relevantes se encarnan en extensiones distinguidas que se corresponden con esas cualidades. Las cualidades están, por consiguiente, diferenciadas, y también lo están las extensiones en cuanto representan líneas divergentes según las cuales se actualizan las relaciones diferenciales que sólo coexisten como Idea. (DELEUZE, 2012, p. 366/2011, p. 315, cursivas nuestras).

de multiplicidades (alloiosis), es así como se nos propone pensar la naturaleza del movimiento (virtual y actual) que define sustancialmente a lo real. 
La idea de color al estar constituida por una coexistencia virtual de relaciones diferenciales de un cierto orden puede ser definible por los componentes heterogéneos que la recorren y que se co-implican en un mismo punto; como hemos visto, la luz blanca concentra virtualmente "la diversidad indefinida de los rayos multicolores”. Y, como propondrá Deleuze, la diversidad es lo dado, y "[...] aquello por lo que lo dado es dado como diverso" no es sino la diferencia (DELEUZE, 2012, p. 333/2011, p. 286). Dicho en otros términos, si el movimiento de lo virtual es inseparable de un proceso de actualización, al situar la diferencia y su concepto en el tiempo, las relaciones diferenciales que actualizan colores cualitativamente distintos se explican por un movimiento de diferenciación inmanente al concepto de diferencia. Por esto, "[c] uando se anula explicándose en lo extenso, la diferencia como intensidad permanece implicada en sí misma." (DELEUZE, 2012, p. $342 / 2011$, p. 294). Pues bien, si el color en tanto concepto (virtual) consiste "en volver sus componentes inseparables dentro de él: distintos, heterogéneos y no obstante no separables" (DELEUZE, 2009, p. 25/2005, p. 25), al ser cada componente del color inseparable dentro de su concepto, la multiplicidad virtual del color concentrada en un punto recorrería "[...] incesantemente sus componentes, subiendo y bajando dentro de ellos. [Y] Cada componente en este sentido es un trazo intensivo, una ordenada intensiva, que no debe ser percibida como general ni como particular, sino como una pura y simple singularidad." (DELEUZE, 2009, p. 25-26/2005, p. 25). Si la diferencia en tanto intensidad permanece implicada en sí misma, y la multiplicidad virtual se actualiza, es porque "[...] toda diversidad, todo cambio remiten a una diferencia que es su razón suficiente. Todo lo que pasa y aparece es correlativo de órdenes de diferencias: diferencia de nivel, de temperatura, de presión, de tensión, de potencial, diferencia de intensidad." (DELEUZE, 2012 , p. 333/2011, p. 286). Dicho en otros términos, si el concepto de color se compone de luz blanca, él también deviene diversidad de matices de colores y, a la vez, permanece implicado en dichos matices por órdenes de diferencias. Podríamos decir, la luz actualiza la diversidad de los colores y permanece virtualmente implicada en los mismos. Miguel de Beistegui, respecto a la concepción hetero-onto-genética de la diferencia en Deleuze ${ }^{6}$, ha descrito la relación virtual y actual del siguiente modo:

${ }^{6}$ El estudio de de Bestegui nos propone aproximarnos al pensamiento deleuzeano considerando las revoluciones científicas que han ocurrido después de Newton. Lo que significaría al menos problematizar nociones clásicas, como las de substancia o permanencia, que atraviesan de distintos modos las historias de la filosofía, al considerar que la materia pensada por las ciencias post-newtoneanas presuponen el devenir de lo real. 
La intensidad es de hecho inseparable de la extensión, de la misma manera que lo problemático es inseparable de su solución: ellos son los dos lados que componen lo real, la imagen del movimiento del ser en cuanto génesis. Ser es esta irreductible composición, intensidad/extensión [Being is this irreducible assemblage, intensity/extensity]. Sin embargo, la intensidad o la diferencia, es anulada por la extensión o la identidad. [...]. Pero es la diferencia en tanto intensidad la que crea el sistema explicándose a sí misma, y la solución no es tanto la negación de la diferencia sino más bien la afirmación de ella, incluso si también la borra. (BESTEGUI, 2004, p. 310 , traducción nuestra).

¿Qué comporta mantener esta diferencia de la diferencia misma? ¿Qué implica considerar el punto virtual de la pura luz blanca $y$ la actualidad de la diversidad de los matices de color? Atendamos a lo siguiente, si Deleuze ha sido llevado "[...] a formar el concepto de diferent/ciación, para indicar a la vez el estado de las relaciones diferenciales en la Idea o la multiplicidad virtual, y el estado de las series cualitativa y extensiva, donde se actualizan diferenciándose." (DELEUZE, 2012, p. 366/2011, p. 316), él mismo planteará que aún permanece como indeterminada la condición de tal actualización (DELEUZE, 2012, p. 366/2011, p. 316). Y el determinarla consistiría en atribuirle un papel determinante a las diferencias en intensidad en tanto factor individuante. Pues bien, quizá no se trate sino de seguir un movimiento de individuación "[...] independiente de la explicación por el orden de implicación que la define [...] independiente de la diferenciación por el proceso que le pertenece esencialmente." (DELEUZE, 2012, p. 367/2011, p. 316-317). Entonces, ¿`cómo el color va implicando su multiplicidad virtual en la actualización del mismo, o sea, cómo concebir un concepto en su desenvolvimiento inmanente?

\section{II}

Esto, como ha señalado Ruiz Stull a propósito de Bergson (2013, p. 44), debe llevarnos a considerar una suerte de arte en la práctica de las distinciones en cuanto esta no podría desconocer su pertenencia al orden de los cortes producidos por el lenguaje, y de lo que se trataría es de atender al movimiento indivisible y heterogéneo de un color que entrelaza lo virtual y lo actual en cuanto su condición es real. Pues bien, si consideramos que las artes en cuanto artificios-sensibles expresan otro plano en que se envuelve y desenvuelve un pensamiento, al ya no 
tratar con conceptos en sus modos de expresión sino con bloques percepto-afectos que tornan consistente la materia en que se juega lo real (¿Qué es la filosofía?, capítulo 7), podríamos atender a determinadas obras que podrían llevarnos a experimentar intensidades de color que las artes hacen visibles y/o audibles y/o legibles. ${ }^{7}$ Es decir, como diría Deleuze y Guattari, al plantearse como tarea la creación de bloques percepto-afectos en un plano de composición distinto al plano de inmanencia (filosófico) y al plano de referencia (científico), las artes buscarían dar expresión a sensaciones que habríamos de calificar de intensas (QPh, capítulo 7). Intensidades que circulan en el campo filosófico al este estar implicado en sus demandas (BERGSON, 2013, p. 44), y esto porque habríamos de concebir interferencias intrínsecas entre dicho planos que nos permitirían concebir un complejo sensación/concepto para seguir elaborando un concepto-sensación de color (DELEUZE; GUATTARI, 2009, p. 218-220/2005, p. 204-206). Ahora bien, antes de volver sobre lo dicho extraeremos una premisa experimental expresada por G.W Goethe en su Teoría de los colores, ya que esta nos permitiría ir de un plano de referencia sostenido por un quehacer científico hacia un plano de composición en el que un ejercicio artístico se elaboraría para luego intentar esbozar una interferencia filosófica. Y creemos que no es menor dicha obra en tanto ocupa un lugar en Deleuze como en Paul Klee. ${ }^{8}$

Si un plano de referencia básicamente delimita un movimiento espaciotemporal al atribuirle determinadas funciones: un sistema de coordenadas (p.j, un plano geométrico euclidiano o no-euclidiano) un estado de cosa (p.j, relación entre partículas o células), cosa (p.j, partícula, célula) una determinada

7 "Desde otro punto de vista, la cuestión de la separación de las artes, de su autonomía respectiva, de su eventual jerarquía, pierde toda importancia. Porque hay una comunidad de las artes, un problema común. En arte, tanto en pintura, como en música, no se trata de reproducir o de inventar formas, sino de captar fuerzas. Incluso por eso es por lo que ningún arte es figurativo. La célebre fórmula de Klee $<<$ no hacer lo visible, sino hacer visible $>>$ no significa otra cosa. La tarea de la pintura se define como el intento de hacer visibles fuerzas que no lo son. [...]. La fuerza está en estrecha relación con la sensación: es preciso que una fuerza se ejerza sobre un cuerpo, es decir, sobre un punto de la onda, para que haya sensación. Pero si la fuerza es la condición de la sensación, ella sin embargo no es sentida, puesto que la sensación $<<\mathrm{da}>>$ cualquier otra cosa a partir de las fuerzas que la condicionan. [...]. ¿Cómo pintar o hacer que se oiga el tiempo? ¿y las fuerzas elementales como la presión, la inercia, el peso, la atracción, la gravedad, la germinación? A veces, por el contrario, la fuerza insensible de tal arte parece más bien formar parte de los $<<$ datos $>>$ de tal otro arte: por ejemplo el sonido, o incluso el grito, ¿¿cómo pintarlos? (Y al revés: ¿`cómo hacer que se oigan los colores?)” en

Gilles Deleuze (2002, 63-64, fr.57-58)

${ }^{8}$ En Deleuze, habríamos de recordar tanto los estudios sobre cine como Francis Bacon. Lógica de la sensación en la que la presencia positiva de Goethe no deja de ser relevante para preguntar por el estatuto de la imagen. En el caso de Klee, la teoría del color goetheana es fuente de inspiración para la elaboración de su concepción. 
magnitud (p.j peso, medida, extensión) (QPh, Capítulo V), en nuestro caso podríamos atender a la polémica inaugurada por Goethe contra Newton al disputarle el principio que da cuenta de la génesis del color. Como dirá Goethe (2008, p. 269/1984b, p. 21):

La teoría que nosotros preconizamos y de la que aquí sólo hablamos, en cuanto que se opone a la newtoniana, también se ocupa de la luz blanca; ella también recurre a condiciones exteriores para en lo sucesivo dedicarse a los fenómenos cromáticos. Pero a estas condiciones le reconoce valor y dignidad [Wert und Würde]. No pretende desenvolver los colores de la luz, más bien lo que busca es convencernos de que el color es al mismo tiempo generado por la luz y por lo que se enfrenta a ella. (Traducción modificada).

Más arriba apelábamos a un determinado movimiento, este iba desde la luz al proceso de actualización de colores. En otros términos, una fuerza ejerce determinada presión en una materia determinándola a expresarse en colores, y en Goethe dicha materia-fuerza se distingue a través de determinados contrastes que corresponden a distintos órdenes de diferencias: "[...] amarillo y azul, acción y desposeimiento, luz y sombra, claridad y oscuridad, fuerza y debilidad, cercanía y lejanía, atracción y repulsión, afinidad por los ácidos y afinidad por los alcális" (GOETHE, 2008, p. 188-189,/1984a, p. 252-253) que se distribuyen relacionalmente; es decir, la actualidad de los colores se debe a órdenes de diferencias a la que le es correlativa una virtual multiplicidad. $Y$ todo indicaba, si seguimos a Bergson-Deleuze, que era necesario concebir dicho movimiento en términos de duración, esto es, los contrastes están implicados en la naturaleza misma de color. Más aún, habríamos de concebir que lo que los diferencia no es sino un movimiento virtual inseparable de una actualización cuya novedad es irreductible. Entonces, cuando Goethe contrasta su teoría con la newtoniana considera que las condiciones que actualizan el fenómeno del color son esenciales para dar cuenta de la naturaleza lumínica del mismo, las incluye en su función. Y para nosotros de lo que se trataría es de tornarnos hacia su concepto.

Es que el método experimental desarrollado por Goethe no fue otro que el intento de atenerse a las condiciones reales que generan una experiencia del color. Ahora bien, si nos permitimos un minúsculo ejercicio lingüístico quizá podríamos decir que el genitivo puede llegar a ser recorrido en al menos dos direcciones. Esto es, una experiencia del color antecedería a la propia experiencia del color. La individualidad de la experiencia no sería sino producto 
de un incesante proceso de individuación, que en nuestro caso podría pensarse como el de un color. Como dirá Deleuze (2007, p. 350/2003, p. 363), en La inmanencia, una vida:

Una vida sólo contiene virtuales. Está hecha de virtualidades, acontecimientos, singularidades, lo que llamamos virtual no es algo a lo que le falte realidad sino algo que está implicado en un proceso de actualización de acuerdo con el plano que le otorga su realidad propia. $\mathrm{El}$ acontecimiento inmanente se actualiza en un estado de cosas y en un estado de vivencia que hacen que ocurra. El propio plano de inmanencia se actualiza en un Objeto y en un Sujeto a los cuales se atribuye. Pero, aunque parezca inseparable de su actualización, el plano de inmanencia es él mismo virtual, mientras que los acontecimientos que lo pueblan son virtualidades.

Podríamos describir las experiencias de color propuesta por Goethe como si se trataran de un movimiento que va tomando consistencia a medida que va alterándose su punto de vista, a medida, y estas son las dimensiones propuestas por Goethe, que va cambiando fisiológica, física y químicamente; a medida, podríamos decir junto a Deleuze, que va relativizando su velocidad o cuantificándose su exposición. Dicho en otros términos, si en la Teoría de los colores encontramos una descripción experimental del fenómeno del color se debe a que el mismo autor alemán establece parámetros referenciales que nos permitirían aproximarnos al color en su actualidad, desde una descripción subjetiva hacia una descripción objetiva. En otros términos, si dicha teoría elaborada por Goethe pertenece al plano de referencia científico, lo naturalmente dado: los colores, suponen para este autor modos distintos de exposición a medida que el punto de vista va variando fisiológica, física y químicamente. Pero debemos decir que Goethe alienta una interferencia entre las ciencias, las artes y las filosofías (GOETHE, 2008, p. 193-201; Farbenlehre, Band 1, p. 259-274). Pues bien, aquí podría practicarse una inflexión que ya está inscrita en su mismo texto al poder llegar a concebirse una disyunción inclusiva entre los modos que van entretejiendo una experiencia fisiológica, física y química. Esto es, se trataría de preguntar por cómo el entrelazamiento virtual y actual de dichas dimensiones las relaciona sin determinarlas a priori. Si en el cuarto capítulo de Diferencia y repetición, titulado Sintesis ideal de la diferencia, Deleuze (2012, p. 276/2011, p. 236) va a proponer que 
[...] una idea es una multiplicidad definida y continua de $n$ dimensiones. [Y] El color, o más bien la Idea de color, es una multiplicidad de tres dimensiones. Por dimensiones es preciso entender las variables o coordenadas de las que depende un fenómeno; por continuidad es preciso entender el conjunto de relaciones entre los cambios de esas variables, [...]; por definición es necesario entender los elementos recíprocamente determinados por esas relaciones, que no pueden cambiar sin que la multiplicidad cambie de orden y de métrica.

¿Podemos describir dichas variables o coordenadas? Con Goethe podemos responder que sí, y estas son: fisiológicas, físicas y químicas. Es decir, cuando se produce el color, cuando la idea se vuelve actual, es porque se está produciendo determinada relación entre aquellas dimensiones que generan cierta experiencia de color. Esto es, dicha experiencia es producto de un conjunto de relaciones entre aquellas dimensiones que al yuxtaponerse actualizan la multiplicidad virtual que compone la idea de color. ${ }^{9}$ Idea que habríamos de considerar virtualmente en cuanto dicho conjunto de relaciones que la definen pueden combinarse de modos muy distintos al punto en que cualquier variación provoca un cambio en dicha multiplicidad. ${ }^{10} \mathrm{Y}$ Goethe expondría algunos de dichos cambios en los experimentos que sostienen su teoría de los colores. Ahora bien, si se produce una síntesis entre dichos elementos habría que atenerse a las variaciones que las mismas introducen. No da lo mismo, por ejemplo, la composición fisiológico de determinado individuo que puede llegar a tener experiencia del color (y esto por supuesto no se reduce a lo humano), ni la composición química que determina el

9 Como dirá Eladio C. P. Craia (2002, p. 139-140): “A multiplicidade não requer a legitimação de uma Unidade que a preceda a fim de se sustentar; seu sistema, pelo contrário, determina a si próprio enquanto co-determinaçáo recíproca de seus elementos diferenciais. Relaçáo do diferente com o diferente quer dizer, entre outras coisas, que tudo é multiplicidade, que nós nos movemos na multiplicidade, que respiramos multiplicidade, que somos multiplicidade. No entanto, isto nada tem de essência ou de fundamento, pois leva em seu seio, como componente relacionável, nada mais que a Diferença".

10 "Las Ideas, las distinciones de Ideas, son inseparables de su tipos de variedades y de la manera en cada tipo penetra en los otros. Proponemos el nombre de perplicación para designar ese estado distintivo y coexistente de la Idea. No porque la <<perplejidad>>, como aprehensión correspondiente signifique un coeficiente de duda, de vacilación o de asombro, ni nada de inacabado en la Idea misma. Se trata, por el contrario, de la identidad de la Idea y del problema, del carácter exhaustivamente problemático de la Idea, es decir, de la manera en que los problemas están objetivamente determinados por sus condiciones a participar los unos en los otros, de acuerdo con las exigencias circunstanciales de la síntesis de las Ideas." [...]. "El problema pertenece al orden del acontecimiento." (DELEUZE, 2012, p. $284,286 / 2011$, p. 242,244$)$. 
proceso de individuación que genera determinada experiencia del color (y esto por supuesto debe llevarnos a considerar la diversidad de seres que componen milieu distintos), condicionando de otro modo la dimensión propiamente física del color (tono, luminosidad, saturación). De ahí que la síntesis no excluya un elemento para incluir a otro sino que incluye en su diferencia un elemento y otro. Y si dicha síntesis no es sino disyuntiva, es el carácter experimental de la misma lo que habríamos de considerar. Francois Zouravichvilli en su Vocabulario Deleuze ha propuesto una entrada a dicho concepto:

Con Deleuze, la noción adquiere un sentido muy distinto: la no-relación se convierte en una relación, la disyunción es una relación [relation]. [...] [E]lla hace pasar cada término en el otro según un orden de implicación recíproca asimétrica que no resuelve ni en una equivalencia ni en identidad de orden superior. [D]istancia entre puntos de vista, indescomponible y desigual a sí a la vez, porque el trayecto no es el mismo en los dos sentidos. (ZOURAVICHVILLI, 2007, p. 100).

Al resaltar que la noción de relación que está en juego en el pensamiento deleuzeano es irreductible a los términos (elementos) implicados, dicha síntesis al mismo tiempo modifica a estos. En nuestro caso, ya volveremos sobre esto, las relaciones entre las dimensiones que determinan la experiencia de color están implicadas asimétricamente. Por supuesto, esto no quiere decir que cualquier relación pueda llevarse a cabo pero tampoco quiere decir que estén a priori limitadas a relacionarse de determinado modo. Es que una síntesis no es sino una relación entre dimensiones (elementos, términos) no equivalentes; o, como diría Zouravichvilli, al ser asimétricas no son sino ajenas a toda "[...] fijación identitaria [...] preservando el devenir o el proceso deseante.” (ZOURAVICHVILLI, 2007, p. 102). Es decir, dichas dimensiones son determinadas por relaciones que componen una multiplicidad virtual que es dable pensar en su dinamismo tiempo-espacial. "Más aún, es la naturaleza en su conjunto, la multiplicidad ramificada de las especies vivas las que testimonian un escalonamiento o una libre comunicación de problemas y divisiones resolventes que remiten en última instancia al ser unívoco como la diferencia.” (ZOURAVICHVILLI, 2007, p. 101). Síntesis disyuntiva sería entonces una relación concebida en un plano en que se pliegan y despliegan multiplicidades, en un plano en el que el movimiento es inmanente a él ${ }^{11}$, y que quizás habríamos de considerar

${ }^{11}$ Podríamos decir en los términos de Gilbert Simondon (2015, p. 174-175): "Si se plantea que el individuo parte como mínimo de un sistema, la relación deviene tan real como el individuo en tanto 
experimentalmente. Si esto es así, la naturaleza de la diferencia sólo podría ser conceptualizable en su devenir y heterogeneidad constitutiva, es decir, la síntesis disyuntiva está implicada en un movimiento de alteración propio a una multiplicidad virtual/actual: dichas síntesis relacionan dimensiones que no podrían considerarse siendo las mismas. En efecto, si la síntesis disyuntiva nos permite pensar de otro modo el concepto de color, tanto las relaciones como las dimensiones fisiológicas, físicas y químicas pueden variar. La diferencia implica disyunción en cuanto genera otros modos de existencia cuyas dimensiones se actualizan en cada caso distintamente. Ahora bien, ¿cuándo es posible concebir una experiencia de color liberada potencialmente de una actualidad que dado determinado hábito se identifica con un sujeto y un objeto? ¿en qué casos podríamos llegar a pensar que un color fuerza a la misma experiencia liberando su potencia facultativa, es decir, produciendo otras síntesis? El mismo Deleuze ha expuesto que, en inspiración kantiana-nietzcheana,

No se debe contar con el pensamiento para sentar la necesidad relativa de lo que piensa, sino por el contrario con la contingencia de un encuentro con lo que fuerza a pensar, para levantar y erigir la necesidad absoluta de un acto de pensar, de una pasión de pensar. Las condiciones de una verdadera crítica y de una verdadera creación son las mismas: destrucción de la imagen de un pensamiento que se presupone a sí mismo, génesis del acto de pensar en el pensamiento mismo. (DELEUZE, 2012, p. 215/2011, p. 181-182).

\section{III}

$\mathrm{Si}$ nos parece relevante considerar que los colores al remitir a una dimensión fisiológica presuponen un cuerpo, es este el que puede experimentar su propia alteración al variar el plano en que se produce una experiencia colorista. Es decir, si, como hemos dicho, proponemos ir del plano filosófico al plano artístico y viceversa, el concebir otra experiencia fisiológica del cuerpo que ya no atiende a su organicidad sino a su modo de experimentación inorgánico implicaría considerar que las artes harían posible experimentar un

ser, que podría abstractamente ser concebido como aislado. El individuo es ser en relación; es centro de actividad, pero esta actividad es transductiva; se ejerce a través y por un campo de fuerzas que modifica todo el sistema en función del individuo y al individuo en función de todo el sistema. [...] [t]oda relación modifica la estructura y todo cambio de estructura modifica la relación, o más bien es relación, pues todo cambio de estructura del individuo modifica su nivel energético e implica por consecuencia intercambio de energía con otros individuos que constituyen el sistema en el cual el individuo ha recibido su génesis". 
cuerpo sin organización ${ }^{12}$, y que quizá podría llevarse a cabo a través de una sensación de color. Como dirán Deleuze y Guattari (2010, p. 163/1980, p. 196), en una de las lenguas de Mil mesetas, aquel cuerpo se opone "[...] a esa organización de los órganos que llamamos organismos"13, aquel cuerpo implica ir en contra de lo que la tradición filosófica ha denominado Uno.

Nosotros respondemos ahora: es el $\mathrm{CsO}$, él es la realidad glaciar en la que se va a formar esos aluviones, sedimentaciones, coagulaciones, plegamientos y proyecciones que componen un organismo -y una significación y un sujeto-. En él los órganos entran en esas relaciones de composición que llamamos organismos. El CsO grita: ¡me han hecho un organismo! ¡me han plegado indebidamente! ¡me han robado mi cuerpo! El juicio de Dios lo arranca de su inmanencia y le hace un organismo, una significación, un sujeto. Él es el estratificado. Como consecuencia, oscila entre dos polos: las superficies de estratificación, sobre las que se pliega, y se somete al juicio, el plano de consistencia, en el que se despliega y se abre a la experimentación. Y si el $\mathrm{CsO}$ es un límite, si nunca se acaba de acceder a él, es porque detrás de cada estrato hay otro estrato, un estrato encajado en otro estrato. (DELEUZE; GUATTARI 2010, p. 164; 1980, p. 197).

Un cuerpo sin órganos sería una experiencia que habría que hacer ${ }^{14}$, ya que mientras esta está plegada a determinados estratos que le atribuyen una significación y una determinada identidad que la arrancan del devenir, una experiencia sería aquella que desplegaría su potencia virtual en un plano

\footnotetext{
${ }^{12}$ Anne Sauvagnargues, ha expresado el concepto de cuerpo sin órganos del siguiente modo: "[dicho concepto] se forma a partir de una teoría de la literatura, al aproximarse a la locura, capaz de hacer emerger una imagen del cuerpo anterior a su individualidad. El acceso a esta corporeidad material, preindividual, intensiva, puede darse en la literatura. El cuerpo sin órgano sirve para pensar la corporeidad y la morfogénesis de los cuerpos sin relacionarlos a un principio unificante exterior: alma, forma, unidad de un organismo, situándose en el nivel de la materia informe, es decir, en el plano de las fuerzas. Artaud lo despliega, él mismo [en su experimentación literaria y vital (agregado nuestro)], en el nivel inmanente de lo material evitando todo principio de unidad exterior, expresando esta corporeidad sin organización a través de una sintaxis sin articulación". (SAUVAGNARGUES, 2009, p. 85 , traducción nuestra).

13 "El CsO hace pasar intensidades, las produce y las distribuye en un spatium a su vez intensivo, inextenso. $\mathrm{Ni}$ es espacio ni está en el espacio, es materia que ocupará el espacio en tal o tal grado, en el grado que corresponda a las intensidades producidas. Es la materia intensa y no formada, no estratificada, la matriz intensiva, la intensidad=O; pero no hay nada negativo en ese cero, no hay intensidades negativas ni contrarias. Materia igual a energía. Producción de lo real como magnitud intensiva a partir de cero.” (DELEUZE; GUATTARI, 2010, p. 158/1980, p. 189).

${ }^{14}$ Ruiz S. ha escrito un notable texto al respecto de la "fórmula: $\mathrm{CsO}$ " titulado: La fórmula del cuerpo sin órganos. Una aproximación bergsoniana a su enunciación” en Trans/Form/Ação, Marília, v. 34, n.1, p.131-148, 2011.
} 
que podría de otro modo abrirse si es interferido por otro plano en el que un bloque percepto-afecto abre un umbral que le permitiría atender a su génesis. Concebirlo materialmente, atender de otro modo a los miles de hábitos que nos componen para preservar el devenir, sería experimentar un límite que no habría sino que hacer. Pues bien, un caso que nos permitirá contemplar un cruce que abra dicha oscilación hacia la experimentación creemos que sería un cuadro de Paul Klee titulado Con el arcoiris (Mit dem Regenbogen) ${ }^{15}$. Es que ya en Klee, y dicho cuadro sería una suerte de pasaje (passage) a su obra, ocurrirían interferencias en las que se produciría una sensación conceptual. Si en ¿Qué es la filosofía? Deleuze y Guattari proponen una distinción concebible entre las tareas de las artes y de las filosofías, diciendo que

Estos universos [los de las artes] no son virtuales ni actuales, son posibles, lo posible como categoría estética (<<un poco de posible, si no me ahogo >>), la existencia de lo posible, mientras que los acontecimientos son la realidad de lo virtual, formas de un pensamiento-Naturaleza que sobrevuelan todos los universos-posibles, lo que no significa decir que el concepto anteceda de derecho a la sensación: incluso un concepto de sensación tiene que ser creados con sus propios medios, y una sensación existe en su universo posible sin que el concepto exista necesariamente en su forma absoluta. (DELEUZE; GUATTARI, 2009, p. 179/2005, p. 168).

En el pensamiento de Klee podemos hallar una descripción de los movimientos de los colores entre tonalidades claras-oscuras, "[...] die farbigen Hell-Dunkel-Bewegungen" (KLEE, 1956, p. 423) ${ }^{16}$, en la que se describe una síntesis, "[...] die Synthese von Hell-Dunkel-Bewegung" (KLEE, 1956, p. 423), producto de un contraste de fuerzas entre temperaturas, operando entre los órganos de composición de movimiento (Bewegungskomposition) que produce determinadas configuraciones de colores. Pues en el caso de Klee se trata de introducir un matiz fundamental, en un tono que no deja de interferir con las ciencias, para pensar en una sensación/concepto de color dentro del terreno de las artes. Es que los contrastes entre temperaturas, una dimensión energética que para Klee (1956, p. 421) va del blanco al negro, "verstehen als Energiesteigerung", relacionan movimientos tonales que se generan

\footnotetext{
${ }^{15}$ Con el arcoiris (mit dem Regenbogen), 1917, colección privada, Suiza. En Paul Klee. Jahre der Meisterschaft 1917 - 1933. Herausgegeben von Roland Doschka. München: Prestel, 2011. Pueden hallarse en internet varias reproducciones digitalizadas, más entradas buscando con el título en alemán. Por ejemplo, http://www.kunstkopie.de/a/paul_klee/mitdemregenbogen191756-2.html.

${ }^{16}$ Ver también, Paul Klee (1997, p.50-51).
} 
experimentando con las relaciones que la esfera cromática expresa. Como dirá nuestro pintor:

Si surge una acción de color sobre la dimensión claro-oscuro (arriba-abajo) se expande el esquema a través de la dimensión de contrastes de temperatura (izquierda - derecha). Las dos dimensiones conjugadas dan dos direcciones para movimiento [Bewegung] y contra-movimiento [Gegenbewegung] [1]. A medida que la combinación entre ellas aumenta o disminuye también surge la dimensión <<atrás-delante>>. Ella evoca a un giroscopio (Kreisel), compuesto de una vertical y una superficie circular. (KLEE, 1956, p. 424).

1.
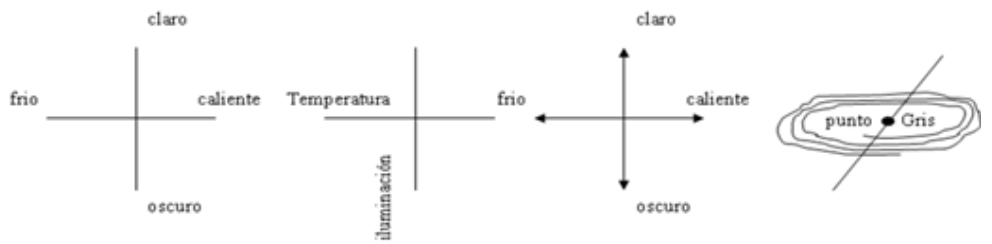

"En la línea circular se efectúa un compacto movimiento sin fin, y ya no hay necesidad de un contramovimiento. Movimiento y contramovimiento se combinan en una centrifuga oscilación pendular [zentrifugalen Pendelschwung] [2]." (KLEE 1956, p. 425).

2.

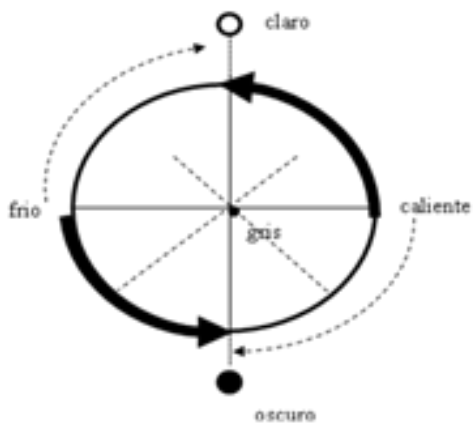


El cinturón con los colores del espectro es por así decirlo el ecuador [3]. Los puntos negro y blanco son los polos [2]. El punto gris se sitúa en equidistancia de los cinco elementos de base: blanco, azul, amarillo, rojo, negro. Los colores se localizan en izquierda-derecha, detrás-delante del plano, y en los bordes de la circunferencia se encuentran más puros. Las relaciones más puras de color se efectúan en las vías periféricas. La última limitación con total equilibrio de balance sería el gris, armonía desprovista de vida. (KLEE, 1956, p. 425, traducción nuestra).

3.

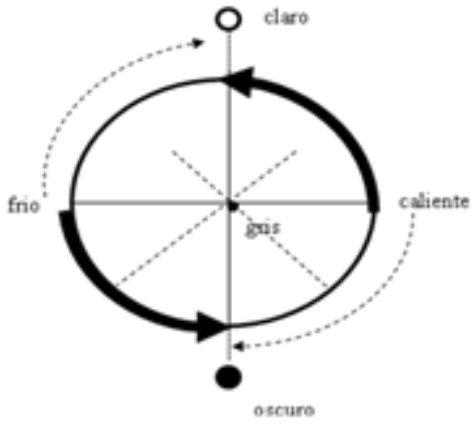

Klee concentrará en un punto la génesis (pictórica) del color implicada en dos dimensiones que al relacionarse conjugan una sensación de color. Ahora bien, para Klee dicha génesis está precedida por el caos: un concepto no-conceptual (unbegrifflicher Begriff) que, "[...] como si del seno del caos se extrajese un resultado", al ser concebible sensiblemente deviene gris: "[...] punto fatídico para el devenir y lo que muere: el punto-gris.” (KLEE, 1956, p. $3)^{17}$. Como dirá Klee (1956, p. 3-4):

Punto-gris es este porque no es ni blanco ni negro o porque es tanto blanco como negro. Es gris porque no está ni arriba ni abajo o porque está tanto arriba como abajo. Gris porque no es ni cálido ni frío, gris en tanto punto

\footnotetext{
${ }^{17}$ Dirá Deleuze citando a Klee en "Los pliegues en el alma” de El pliegue (Barcelona: Paidós, 1989): "el punto como <<concepto no conceptual de la no-contradicción >> recorre una inflexión. Es el propio punto de inflexión, allí donde la tangente corta la curva. Es el punto pliegue" (25 (fr. 20). Este acontecimiento, antes de efectuarse (corte potencial), considerado en su idealidad, va a ser expresado por Klee como: "<<lugar de la cosmogénesis >>, <<punto no-dimensional >>, <<entre las dimensiones >> (DELEUZE, 1989, p. 26/1988, p. 21). En otro decir: génesis del color, caoscatástrofe y germen: punto gris.
} 
no-dimensional, punto entre las dimensiones. El momento cosmogenético está ahí. Al establecimiento de un punto en el caos, punto que, concentrado en principio, solo puede ser gris, se le concede un originario carácter concéntrico. De hecho, de él despierta el orden irradiando hacia todas las dimensiones. Elevar un punto a tal valor central significa concederle el momento cosmogenético [kosmogenetischen Moment]. A este proceso corresponde la idea de cualquier tipo de comienzo (por ejemplo, el embarazo) o mejor aún, el concepto de huevo [BegriffEi]. (Taducción nuestra). ${ }^{18}$

Este valor central atribuido al gris, interferencia entre un planteamiento filosófico del color y uno de corte artístico, promueve un movimiento cosmogenético no ajeno a un acontecimiento; implica, para Klee, atender de otro modo a la génesis del color. Pues bien, si al comenzar a concebir un concepto de color nos remitíamos a una lectura de Deleuze de Bergson para atender a la génesis de su naturaleza concentrada en un único punto: la pura luz blanca, y si con Goethe buscábamos resaltar que esta era también producto de un contraste esencial a ella misma, en Klee dicho punto deviene artístico. El momento cosmogenético (kosmogenetischen Moment) de una obra de arte pictórica no es sino expresable por el punto-gris. Es que al considerar que el movimiento de la naturaleza es esencial para atender al arte de formar, ya que "[...] puede mover al artista desde el fondo y, vuelto móvil el mismo, llegar ahora a ocuparse del libre desenvolvimiento de sus propias composiciones en camino (Gestaltungswegen)." (KLEE, 1956, p. 93), Klee atiende a un problema genético al llevar al plano de experimentación artística un concepto no-conceptual (unbegrifficher Begriff) confrontable percepto, afectiva y filosóficamente. En este sentido, le confiere a las artes una tarea similar al de la naturaleza que no es otra que la creación. En efecto, al promover por decirlo así un nuevo credo en las artes que se sintetiza en la fórmula: "[...] el arte no reproduce lo visible, sino hace visible" (KLEE, 1956, p. 76) que retomada por Deleuze (2009, p. 63/2002, p. 57) deviene: “[...] hacer visible fuerzas que no lo son”, demanda al arte, en el decir de DeleuzeGuattari, componer desterritorializaciones que no van a dejar de expresar una línea de fuga cuyo plano está impelido por un afuera ${ }^{19}$; de ahí que la oscilación sea fundamental y en el caso de Klee esta adviene bajo la figura de un péndulo en cuya problematización no hay sino una línea en juego. En la conferencia acerca

\footnotetext{
${ }^{18}$ Ver también Klee ([19--i], Bildnerische Gestaltunglehre I.1/14-16).

${ }^{19}$ En torno a un sentido general de línea y, en particular, al peligro que concierne a la línea de fuga, ver de "9 na Meseta: 1933. Micropolítica y segmentariedad" en Mil Mesetas, (DELEUZE; GUATTARI, 2010, p. 225-234/1980, p. 271-283). Habremos de recordar que para Paul Klee la noción de línea juega un rol fundamental en la descripción de su propia obra. Ya veremos esto último
} 
del acto de creación, Deleuze consideraba arte, ciencia y filosofía bajo un mismo criterio: "[...] un límite común [...], a saber, la constitución de espacio-tiempos" (DELEUZE, 2007, p. 283/2003, p. 293-294), pues si dichas disciplinas, como lo acentuarán Deleuze-Guattari en ¿Qué es la filosofía?, luchan contra el caos, sus modos de confrontación tendrán relación con cómo hacen frente al problema de la inmersión en él al lidiar con movimientos que cobran determinada relevancia al ser distintamente concebibles en un acto de creación que no podría sino surgir del enfrentamiento con el caos.

Ahora bien, si para pensar en dicho criterio deleuzeano atendemos a la idea de color en su encuentro con una sensación promovida por Klee, en nuestro pintor se trataría de un movimiento en un plano de composición que también implicaría su otra naturaleza, esto es, buscaría atender a su diferencia germinal y al caos contra el que lucha. Con el arcoíris (Mit dem Regenbogen) produciría una interferencia entre planos al concebir que la naturaleza del arcoíris no es suficiente para atender a las fuerzas coloristas que tornan visible una pintura. En dicha pintura se relacionan líneas-fuerzas y al mismo tiempo hace visible otras fuerzas, desterritorializa determinada presentación del color al experimentar con él artísticamente. Como hemos visto, las figuras que han descrito los movimientos de tonos y de temperatura que originan el color componen un plano que configura un círculo. Y esto se debería a que al contrastar la figura del arcoíris, Klee (1956, p. 469-472) va a concebir que

Podemos, por tanto, tranquilamente llamar al arcoíris una representación lineal de los colores y al mismo tiempo una representación insuficiente de estos, de ella podemos aprender bien poco y sobre la relación entre los colores incluso nada. [...] La principal falencia consiste en la finitud de estas series de color. Los colores puros son un asunto del más allá [enseitige Angelegenheit]. El reino intermediario atmosférico es muy bueno para transmitírnoslo, pero es una forma intermedia y no nos la transmite en su forma de más allá cuya naturaleza debe ser infinita [die unendlicher Natur sein muß]. [...] El asunto cósmico de los colores puros ha encontrado su apropiada representación en el círculo. La aparición terrestre y distinguible de los colores puros en el arcoíris no era sino un mero reflejo de una totalidad desconocida, que ahora nosotros extendemos en su forma sintética y la hacemos corresponder al gran Todo. Ante nuestros ojos ahora está el círculo cromático.

Si contemplamos de cerca esta novedosa forma de presentar colores, esto es, el círculo cromático, podríamos distinguir con interés cuán capaz es esta nueva forma de enseńarnos sobre las relaciones de los colores entre 
ellos. En primer lugar, los enigmas de la serie finita de colores están resueltos, o ya no nos preguntamos por ellos. La escala dada por el violeta ya no hay que adivinarla, y ya no nos enfrentamos a tales problemas de localización como el del infra-rojo o el ultra-violeta [los dos extremos del arcoíris]. El nuevo movimiento se ha tornado ahora en un asunto de la circunferencia del círculo, y me gustaría darle el nombre de movimiento periférico, asimilable a un entrelazado intercambio infinito. Este es uno de los aspectos de la novedad. El otro se revela a nosotros en los tres diámetros con los cuales pueden relacionarse los seis colores y dividirse en tres pares de colores [4]. (Traducción nuestra). ${ }^{20}$

4 .

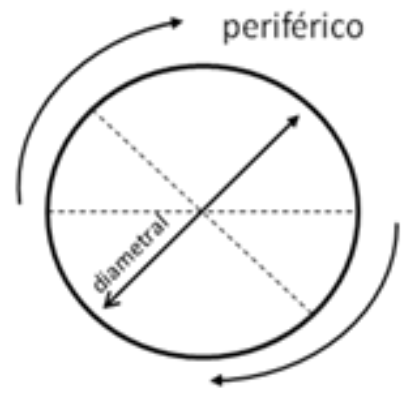

Klee, al experimentar con la experiencia del color expresa las fuerzas que subyacen a él. Si el círculo cromático deviene expresión de "más allâ", se debe a la fórmula artística que Klee desenvuelve al proponer una ruta que problematiza los límites que presentan al visibilizarse. Si volvemos entonces al planteamiento fisiológico en un entrecruce filosófico y artístico, considerándolo en su proceso de individuación, intentemos atender a los miles de hábitos que constituyen una determinada organización en otra exposición de ella, en otro desarreglo de su sintaxis, en una microscópica expresión. Como dirá Deleuze (1989, p. 115/1988, p. 117) en El pliegue:

Debemos comprender literalmente, es decir, matemáticamente, que una percepción consciente se produce cuando dos partes heterogéneas, por lo menos, entran en una relación diferencial que determina una singularidad. [...] Supongamos el color verde: por supuesto, el amarillo y el azul pueden ser percibidos, pero, si su percepción desaparece a fuerza de devenir

${ }^{20}$ Ver también, "Beiträge zur bildnerischen Formlehre" en Paul Klee (1987, p. 266-274). 
pequeña, entran en una relación diferencial [daz/dam] que determina el verde. Y nada impide que el amarillo, o el azul, cada uno por su cuenta, no esté ya determinado por la relación diferencial de dos colores que nos escapan, o de dos grados de claroscuro: $d y / d x=$ AM. [...] La $<<$ buena forma $>$ macroscópico siempre depende de procesos microscópicos. Toda conciencia es umbral.

"Somos agua, tierra, luz y aire contraídos, no sólo antes de reconocerlos o de representarlos, sino antes de sentirlos. Todo organismo es, en sus elementos receptivos y perceptivos, pero también en sus vísceras, una suma de contracciones, de retenciones y de esperas." (DELEUZE, 2012, p. 123/2011, p. 99). Si entre dichas contracciones, entre dichas retenciones y dichas esperas, se altera un flujo, modificar la <<buena forma >> macroscópica no sería sino intentar hacerla devenir otros procesos microscópicos; otras combinaciones, otros umbrales de conciencia. Si el círculo cromático expresa una ilimitada experiencia de color en tanto los colores pueden expresarse de modo infinito; "visibilizar fuerzas que no son visibles" ejemplo, la fuerza del tiempo, consistiría en hacer de un color una potencia artística cuyo revuelo estaría retornando cada vez que se expone una sensación producto de una combinación de fuerzas que nace de un enfrentamiento contra el caos. En el caso del pintor Klee, un bloque percepto-afecto es posible de crear a través de los elementos específicos y formales de la pintura: "[...] la línea, tonalidades de claro-oscuro y color, que a pesar de sus diferencias fundamentales estos elementos plásticos atinentes a la extensión, al peso y a la cualidad mantienen entre sí ciertas relaciones." (KLEE, 1979, p. 19). Pues al concebir que dichos elementos configuran direcciones y dimensiones, ellos crean otros cuadros de experiencia. Es que creemos, para comenzar a concluir, que de Klee a Deleuze habría un movimiento por recorrer. Como dirá nuestro pintor en su credo creativo:

Todo devenir yace en movimiento [Bewegung liegt allem Werden zugrunde]. En el Laocoonte, en el que en nuestra juventud malgastamos no poco esfuerzo intelectual, Lessing hizo de la distinción entre arte del tiempo y arte del espacio toda una preocupación. Pero mirándola de cerca es tan solo una erudita ilusión. Pues el espacio es también una noción temporal.

Se requiere del tiempo para que un punto se torne línea y movimiento. Otro tanto, cuando una línea engendra un plano al desplazarse. Y del mismo modo, para el movimiento que lleva de los planos hacia los espacios. 
¿Acaso una obra pictórica se genera instantáneamente? No, ella se construye pieza por pieza, tal como una casa. [...]

La obra pictórica nace del movimiento, ella misma es movimiento fijado, y se torna experimentable en movimiento [músculos de los ojos (Augenmuskeln)].

La relación del arte respecto a la creación es simbólica. El arte es en cada caso un ejemplo, tal como la tierra es un ejemplo del Cosmos.

La liberación de los elementos, su arreglo en subdivisiones complementarias, la disección y reconstrucción simultánea en dirección al todo bajo diversos ángulos, la polifonía pictórica, la producción de equilibrio a través de compensaciones de movimiento: todo esto son problemas de forma, decisivos para el saber formal, pero no todavía arte en su sentido supremo. Detrás de la ambigüedad de este sentido yace un secreto último, y en este estadio, la luz del intelecto [das Licht des Intellekts] funestamente expira.

Pues a la larga incluso la realidad intensificada no puede pervivir largo tiempo. ¡El arte juega con las realidades últimas un desconocido juego y sin embargo efectivamente las alcanza! (KLEE, 1956, p. 78-80, traducción nuestra).

Bien podríamos considerar que se trataría de proponer un movimiento, y si la dirección comienza a bifurcarse en al menos dos sentidos: desde Klee hacia Deleuze, desde Deleuze hacia Klee, quizá el movimiento de un color pueda ser problematizado de distinto modo. Para nosotros sería un paso considerar que podría pensarse en otros casos en cuanto que al hacer de sus compontes inseparables una sensación podría generar una línea de experimentación en la que una fuga estaría en juego. Y si, como decía Deleuze y Guattari, una sensación es heterogénea a un concepto, y viceversa, y esta diferencia pertenecen a ambos planos distintamente, lo que podría problematizarse sugiriendo una interferencia que hemos intentado elaborar tomando al color como ejemplo no sería sino un movimiento de desterritorialización. Ahora bien, si un concepto de color se torna un asunto cuya complejidad radica en concebir su acontecimiento y no su significado, la heterogeneidad nos anunciaría aún más el concepto inconceptualizable (unbegrifflicher Begriff) del cual provendría. Esto es, un color, y la línea abstracta que lo desterritorializa, quizá no habría sino que como componente de un caos-cosmos: un caos contra el cual se lucha para componer un cosmos. Como diría una fórmula multinómica Klee-Deleuze-Guattari: 
No son tres momentos sucesivos en una evolución. Son tres aspectos de una sola y misma cosa, el Ritornelo. Se encuentra en los cuentos, de terror o de hadas, y también en los lieder. El ritornelo presenta los tres aspectos, los hace simultáneos, o los combina: sea, sea, sea. Sea el caos un inmenso agujero negro, y uno se esfuerza en fijar en él un punto frágil como centro. Sea que uno organiza alrededor del punto una "andadura" (más que una forma) tranquila y estable: el agujero negro ha devenido una casa. Sea que uno introduce en esa andadura una salida, fuera del agujero negro. Paul Klee es quien más profundamente ha mostrado esos tres aspectos, y su relación. Él habla de "punto gris", y no de agujero negro, por razones pictóricas. Pero el punto gris es en primer lugar el caos no dimensional, no localizable, la fuerza del caos, manojo enmarańado de líneas aberrantes. Luego el punto "salta por encima de sí mismo" y hace irradiar un espacio dimensional, con sus capas horizontales, sus capas verticales, sus líneas habituales no escritas, toda una fuerza interna terrestre (esta fuerza también aparece, con una andadura menos enmarańada, en la atmósfera o en el agua). El punto gris (agujero negro) ha cambiado, pues, de estado, y ya no representa el caos, sino la morada o la casa. Por último, el punto se lanza y sale de sí mismo, bajo la acción de fuerzas centrífugas errantes que se despliegan hasta la esfera del cosmos: "Para despegar de la tierra hay que realizar un esfuerzo por impulsos, pero al siguiente nivel uno se eleva realmente por encima de ella [... bajo el imperio de fuerzas centrífugas que triunfan sobre la gravedad". (Mil mesetas, 11va Meseta. 1837. Del Ritornelo, p. 318-319, 383, apud KLEE, 1985, p. 55, 27/2007, p. 55, 28).

\title{
Una composición que no habríamos solo de intentar ver, sino también de escuchar. Un movimiento de desterritorialización por explorar.
}

MARTÍNEZ V., S. Deleuze, color, and cosmos-Klee. Trans/form/ação, Marília, v. 41, n. 1, p. 223-248, Jan./Mar., 2018.

\begin{abstract}
In this work we seek to propose a theory of color through Deleuzian inspiration; that is, we will propose a theory that looks on color as multiplicity and event. We therefore practice a route through some Deleuzian passages that will allow us to move forward in this proposal and take it to the territory of art. We do this in order to establish an interference that will change the direction of our work: we propose a dialogue with Paul Klee. In short, we attempt to develop the following questions: how can we think of a theory of color through Deleuzian inspiration? How can we take it to the territory of art? How can we conceive of its relation to a cosmos-Klee? In discussing these questions, we intend to open another perspective on the work of Gilles Deleuze.
\end{abstract}

KeYwords: Deleuze, Klee, Color, Multiplicity, Event, Arts. 


\section{REFERENCIAS}

BEISTEGUI, M. Onto-hetero-genesis: thinking difference with Deleuze. In: Truth and génesis: philosophy as differential ontology. Bloomington: Indiana University Press, 2004. p. 185-334.

BERGSON, H. El pensamiento y lo moviente. Traducción Pablo Ires. Buenos Aires: Cactus, 2013.

BRYANT, L. R. Difference and givennes. Illinois: Northwestern University Press, 2008.

CRAIA, E. C. P. A problemática ontológica em Gilles Deleuze. Paraná: Editora e Gráfica Universitária; EDUNIOESTE, 2002.

DELEUZE, G. El pliegue: Leibniz y el barroco. Traducción José Vásquez Pérez y Umbelina Larraceleta. Barcelona: Paidós, 1989. (Original) Le pli: Leibniz et le baroque. Paris: Les Éditions de Minuit, 1988.

. La concepción de la diferencia en Bergson. In: . La Isla Desierta y otros textos: textos y entrevistas (1953-1974). Traducción José Luis Pardo. Valencia: Pre-Textos, 2005. p. 45-69. (Original) La conception de la différence chez Bergson. In: L'Tle Déserte: textes et entretiens 1953-1974. Édition préparée par David Lapoujade. Paris: Les Éditions de Minuit, 2002. p. 43-72.

- ¿Qué es el acto de creación? Textos y entrevistas (1975-1995). Traducción José Luis Pardo. Valencia: Pre-Textos, 2007. (Original) Qu'est-ce que l'acte de création? In: . Deux régimes de fous. Textes et entretiens 1975-1995. Édition préparée par David Lapoujade. Paris: Les Éditions de Minuit, 2003. p. 291-302.

. La inmanencia: una vida... In: . Dos regimenes de locos: textos y entrevistas (1975-1995). Traducción José Luis Pardo. Valencia: Pre-Textos, 2007. p. 347-351. (Original) L'immanence: une vie... In: Deux Régimes de fous: textes et entretiens 1975-1995. Édition préparée par David Lapoujade. Paris: Les Éditions de Minuit, 2003. p. 359-363.

- Diferencia y repetición. Traducción María Silvia Delpy y Hugo Beccacece. Buenos Aires: Amorrortu, 2012. (Original) - Différence et répétition. Paris: Presses Universitaires de France, 2011.

. Francis Bacon: lógica de la sensación. Traducción Isidro Herrera. Madrid: Arena, 2009. (Original) . Francis Bacon: Logique de la sensation. Paris: Éditions du Seuil, 2002.

.; GUATTARI, F. Mil mesetas: capitalismo y esquizofrenia. Traducción José Vásquez Pérez, con colaboración de Umbelina Larraceleta. Valencia: Pre-Textos, 2010. (Original) . Mille plateaux: capitalisme et schizophrénie. Paris: Les Éditions de Minuit, 1980.

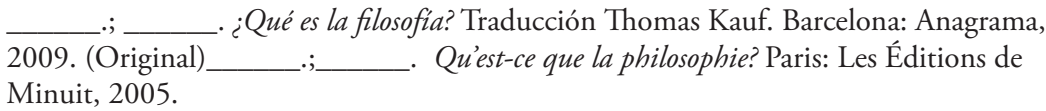


DOSCHKA, R. Paul Klee: Jahre der Meisterschaft 1917 - 1933. München: Prestel, 2011.

GOETHE, J. W. von. Farbenlehre Band 1. Herausgegeben von Ott, Gerhard und Proskauer, Heinrich O. Stuttgart: Freies Geistesleben, $1984 \mathrm{a}$.

. Farbenlehre. Band 3. Herausgegeben von Ott, Gerhard und Proskauer, Heinrich O. Stuttgart: Freies Geistesleben, $1984 \mathrm{~b}$.

- Teoría de los colores. Traducción Pedro Simón. Valencia: Consejo General de la Arquitectura Técnica de España, 2008.

KLEE, P. Das bildnerische Denken. Herausgegeben und bearbeitet von Jürg Spiller. Basel: Benno Schwabe \& Co, 1956.

. Kunst-Lehre. Ausgewählt und herausgegeben von Günther Regel. Leipzig: Philipp Reclam, 1987.

. Bildnerische Gestalungslehre. [19--j]. Disponible en: <http://www.

kleegestaltungslehre.zpk.org/>. Acceso en: 10 jun 2015.

. Théorie de l'art moderne. Édition et traduction établies par Pierre-Henri

Gonthier. Paris: Denoël, 1985.

. Teoría del arte moderno. Traducción Pablo Ires. Buenos Aires: Cactus, 2007.

LORD, B. Deleuze and Kant. In: SMITH, D. W.; SOMERS-HALL, H. (Ed.). The Cambridge Companion to Deleuze. Cambridge: Cambridge University Press, 2012. p. 82-102.

MONTENEGRO, G. Empirismo trascendental: génesis y desarrollo de la filosofía de Gilles Deleuze. Bogota: Editorial Universitaria, 2013 (Serie Filosófica, n. 23).

RÖLLI, M. Deleuze on intensity differentials and the being of the sensible. Deleuze Studies, v. 3, n. 1, p. 26-53. 2009.

RUIZ STULL, M. La fórmula del cuerpo sin órganos: una aproximación bergsoniana a su enunciación. Trans/Form/Ação, Marília, v. 34, n. 1, p. 131-148, 2011.

. Experiencia y empirismo trascendental. In: DIAZ, E. (Ed.). Gilles Deleuze y la ciencia. Buenos Aires: Biblos, 2013a.

- Tiempo y experiencia: variaciones en torno a Bergson. Prologada por Pablo Oyarzún. Chile: Fondo de Cultura Económica, 2013b.

SAUVAGNARGUES, A. Deleuze et l'art. Paris: Presses Universitaires de France, 2009a.

Deleuze, l'empirisme transcendantal. Paris: Presses Universitaires de France, 2009b.

SIMONDON, G. La individuación: a la luz de las nociones de forma y de información. Traducción Pablo Ariel Irs. Buenos Aires: Cactus, 2015.

SMITH, D. W. Deleuze's theory of sensation: Overcoming the Kantian Duality. In: PATTON, P. (Ed.). Deleuze: A Critical Reader. Oxford: Blackwell, 1997. p. 29-56. 
MARTÍNEZ V., S.

ZOURABICHVILI, F. Deleuze: una filosofía del acontecimiento. Traduccon Irene Agoff. Buenos Aires: Amorrortu, 2004. 2007.

El vocabulario de Deleuze. Traducción Víctor Goldstein. Buenos Aires: Atuel,

Recebido: 08/09/2015

Aceito: 18/07/2016 